\title{
Fatty Acids and Triacylglycerols Profiles from Sicilian (Cold Pressed vs. Soxhlet) Grape Seed Oils
}

\author{
Vita Di Stefano ${ }^{1, *}{ }^{\mathbb{C}}$, David Bongiorno ${ }^{1}$, Carla Buzzanca ${ }^{1}$, Serena Indelicato ${ }^{1}$, Antonello Santini ${ }^{2}{ }^{\mathbb{D}}$,

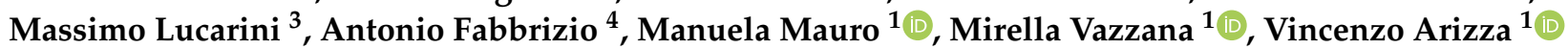 \\ and Alessandra Durazzo ${ }^{3}$ D
}

1 Department of Biological, Chemical and Pharmaceutical Science and Technology (STEBICEF), University of Palermo, Via Archirafi, 90123 Palemo, Italy; david.bongiorno@unipa.it (D.B.); carla.buzzanca@gmail.com (C.B.); serena.indelicato@unipa.it (S.I.); manuela.mauro01@unipa.it (M.M.); mirella.vazzana@unipa.it (M.V.); vincenzo.arizza@unipa.it (V.A.)

2 Department of Pharmacy, University of Napoli Federico II, Via D. Montesano 49, 80131 Napoli, Italy; asantini@unina.it

3 CREA-Research Centre for Food and Nutrition, Via Ardeatina 546, 00178 Rome, Italy; massimo.lucarini@crea.gov.it (M.L.); alessandra.durazzo@crea.gov.it (A.D.)

4 University eCampus, Novedrate, 22060 Como, Italy; antfab13@gmail.com

* Correspondence: vita.distefano@unipa.it; Tel.: +39-2389-1948

check for updates

Citation: Di Stefano, V.; Bongiorno, D.; Buzzanca, C.; Indelicato, S.; Santini, A.; Lucarini, M.; Fabbrizio, A.; Mauro, M.; Vazzana, M.; Arizza, V.; et al. Fatty Acids and Triacylglycerols Profiles from Sicilian (Cold Pressed vs. Soxhlet) Grape Seed Oils. Sustainability 2021, 13, 13038. https://doi.org/10.3390/su132313038

Academic Editor: Dario Donno

Received: 28 October 2021

Accepted: 22 November 2021

Published: 25 November 2021

Publisher's Note: MDPI stays neutral with regard to jurisdictional claims in published maps and institutional affiliations.

Copyright: (c) 2021 by the authors. Licensee MDPI, Basel, Switzerland. This article is an open access article distributed under the terms and conditions of the Creative Commons Attribution (CC BY) license (https:/ / creativecommons.org/licenses/by/ $4.0 /)$.

\begin{abstract}
Among the Sicilian economic productive sectors, that of wine production has today a considerable economic value. However, with the growth of this sector, notable was the increase in the production of waste, which to date is not only an economic damage for companies, but also a threat to the environment. It is known that waste from wine production has properties (e.g., antioxidants) which have potential reuse at cosmetic, pharmaceutical and nutritional levels to obtain economically sustainable applications. A new goal is given by the recovery of added value compounds from agri-food wastes and by-products. Grape seed oil is a promising vegetable fat and cold pressing does not involve the use of chemicals, which are harmful to health. It implies that cold-pressed seed oils may contain phytochemicals, as well as natural antioxidants, more than refined oils. In this context, this works aims at studying the chemical characterization (triglycerides profile and composition in fatty acids) of grape seed oils obtained from Soxhlet and cold pressed extraction from Sicilian red grape seeds and white grape seeds. The possibility of obtaining high yields of triglycerides and fatty acids from the waste of wine production through new extraction methods would open up new perspectives for the reuse of waste in a human and animal food context. The results of this work allow the opening up of new perspectives to reuse and then reduce these wastes, helping not only to reduce the damage to the environment and costs for companies but also to create a new product that is environmentally sustainable and with an important economic value.
\end{abstract}

Keywords: grape seed oil; fatty acids; biorefinery; circular economy; Soxhlet extraction; cold pressed extraction

\section{Introduction}

Currently, food waste is generated in great quantities worldwide: for each type of food, different percentages of food waste are generated along food chain and industrial processing [1]. The "Universal Recovery Strategy", in the perspective of biorefinery and the circular economy concept, represents a new challenge for the commercial recovery of added-value compounds for different applications such as food, feed, cosmetics, biomedical and agronomic ones [2,3].

The residues produced by the various supply chains are characterized by a high intrinsic value given the content of bioactive molecules which, properly extracted and purified, could be reintroduced in different production cycles. The development and 
innovation of technologies for the recovery of molecules of nutritional/nutraceutical interest from by-products and waste of agri-food and industrial production chains has the dual objective of recovering otherwise unused or underused valuable components and favoring the development of targeted innovative supply chains to the strengthening of the local/circular economy.

New trends in food sector research are oriented to give new value to the wastes of some agricultural production processes. In this context is interesting the case of grapes in the vinification process that produce a huge amount of waste [4,5]. The wine world market is dominated by Italy, France and Spain, which together cover more than 50\% of world production [6]. Winemaking produces a series of by-products, easily exploitable for new productions and in various supply chains. Each grape contains from 1 to 4 seeds, the so-called grape seeds, rich in oil with omega 6 fatty acids and other bioactive compounds with antioxidant activity. Once the grape fermentation process is finished and the wine has been extracted, usually the discarded part of the must, the pomace, is eliminated without any reuse. In the case of grape seed oil, on the other hand, it becomes a precious raw material to obtain, following the rinsing of the seeds and an extraction process, the final oil product.

Grape seed oil, a by-product of the wine industry, if recovered from winemaking waste is therefore a symbol of sustainability and zero waste and reflects a virtuous model of the "circular economy". There are different methods for extracting oil from grape seeds. The most common and most commercial is certainly the hot method with the use of solvents, capable of obtaining a higher yield at the expense of the healthful properties of the oil, which in this way completely loses its polyphenol and antioxidant content [7-9]. On the other hand, the cold extraction method, of pure and simple pressure, is much healthier, with significantly lower yields but an extremely higher quality of finished product and which can result in a higher content of fatty acids and tocopherols in the final product [10-12]. The grape seeds oil yields also depend on other factors such as the environmental conditions of the harvest year, the grape variety used, the soil types and so on. However, the grape seeds contain a percentage of oil ranging from $6 \%$ (obtained from black varieties such as Cinsaut and Gamay) to $20 \%$ (from varieties of white sweet grape seeds such as Isabella and Muscat of Hamburg) per $100 \mathrm{~g}$ of dry weight [13-16]. It is interesting that, in their composition, hydrophilic compounds, which include phenolic acids, flavonoids, tannins, carotenoids, tocopherols and stilbenes, were known for their bioactive properties. These substances are, however, present in the oil at a low percentage. The main constituents of the oil are triacylglycerols (TAGs) that, conversely, are constituted by fatty acids (FA) whose main contributor is linolenic acid, progenitor of the omega-6 series. Linoleic acid percentages range from $66 \%$ to $70 \%$ of total fatty acids. Precisely with regard to the content of these essential fats, the product is recognized for its excellent ability to lower blood triglycerides and among the most effective cholesterol-lowering properties, thus decreasing the levels of total cholesterol in the blood, especially the "bad" LDL (low-density lipoproteins) type. Oleic acid is the main mono-unsaturated fatty acid with average concentrations of $15 \%$ of total fatty acids, while among the saturated fatty acids, palmitic is the main one with a variable percentage between $7-8 \%$ of total fatty acids. It is important to underline that the consumption of oil rich in omega 6 must be taken into consideration and according to nutritionists, the daily intake of omega-6/omega-3 should not exceed the 4/1 ratio [17,18]; in fact, an unbalanced assumption of omega- 6 could lead to the formation of harmful eicosanoids. The use of grape seed oil has also been tested as a supplement in diets of animals intended for human consumption. Following the integration of grape seed oil into the animal diet, reductions in lipid content and cholesterol content have been demonstrated in chickens and pigs [8,19], while in ruminants, supplementation with grape seed oil did not lead to positive changes in the lipid profile of the meat [20].

In this context, the aim of this research was to verify how the extraction method of Sicilian grape seed oils (Soxhlet and cold extraction) influences the profile of fatty acids, their relative percentage and triglyceride composition. To corroborate the results on the 
composition of triacylglycerols, a cross-validation of the fatty acid and triacylglycerol profiles was also performed by means of an in-silico saponification software [21].

\section{Materials and Methods}

\subsection{Solvents and Reagents}

Methanol, chloroform, n-hexane, acetic acid, potassium hydroxide and Supelco 37FAME 79248 mix were purchased from Merck (Darmstadt, Germany). HPLC-grade water was obtained by purifying double distilled water in a Milli-Q Gradient A10 system (Millipore, Bedford, MA, USA).

\subsection{Grape Seed Samples}

This study was conducted with different seed grapes of Vitis vinifera L. In detail, red grape seed samples were composed by only one grape's variety, the Sangiovese, while the white grape seeds were a mixture of different grape varieties: Trebiano, Catarratto, Insolia and Grillo $(25 \%, 20 \%, 20 \%$ and $25 \%$, respectively). Wine grape varieties used grew up in western Sicily (Italy) from organic cultivations from the provinces of Trapani, Agrigento and Palermo (100-250 m above sea level) in sunny conditions, mild temperatures and moderate ventilation.

Grapes were harvested in September 2020 at technological maturation, pressed to obtain the juice for production of wine.

Waste products composed of pomace, seeds and grape stems were collected, sieved several times using a large mesh net to recover only the grape seeds, which were dried at $24{ }^{\circ} \mathrm{C}$ for four days (drying step) to reduce the humidity.

\subsection{Sample Treatment}

Grape seed lipids were extracted from dried seeds, obtained after drying step, in two different ways: Soxhlet and cold pressed extraction.

Soxhlet extraction: $30 \mathrm{~g}$ of grape seed were pulverized into fine powders using an electric blender. Oils were obtained by continuous extraction in Soxhlet apparatus for $8 \mathrm{~h}$ using $300 \mathrm{~mL}$ of $\mathrm{n}$-hexane $\left(50-60^{\circ} \mathrm{C}\right)$ as solvent. The solvent was removed with a rotary vacuum evaporator at $40^{\circ} \mathrm{C}$. The separated oil was kept in colored glass bottles at $4{ }^{\circ} \mathrm{C}$ until analysis for fatty acids and triacylglycerols.

Extraction experiments were performed in triplicates and total extraction yield was expressed as mean.

Cold pressed extraction: $3 \mathrm{Kg}$ of grape seeds were put gradually in Cgoldenwall CAN-684 cold press apparatus for extraction of the oil. The oil samples obtained were centrifuged at $4200 \mathrm{rpm}$ for $10 \mathrm{~min}$ at $25^{\circ} \mathrm{C}$ to recover the pure oils and used for fatty acids and triacylglycerols analysis.

For fatty acid analysis, $0.1 \mathrm{~g}$ of sample oil was diluted in $1 \mathrm{~mL}$ of $\mathrm{n}$-hexane and placed in test tubes. A measure of $0.1 \mathrm{~mL}$ of freshly prepared transesterification reagent ( $\mathrm{KOH} / \mathrm{Methanol}, 2 \mathrm{M})$ was added to each tube. The tubes were vortexed for $4 \mathrm{~min}$. Two phases were formed: the upper one (hexane) was transferred to another tube. A measure of $100 \mu \mathrm{L}$ of hexanic phase were added to $900 \mu \mathrm{L}$ of n-hexane and the final (at about 1\%) sample was injected within $6 \mathrm{~h}$ into GC-MS instrument. Three replicate analyses were performed for each grape seeds oil sample.

\subsection{GC-MS Method}

A Thermo Trace DSQ GC-MS (Thermo Scientific ${ }^{\mathrm{TM}}$, Waltham, MA, USA) was used for fatty acid analysis. For FAME analysis (solvent comparison experiment), the MS was operated in positive electron ionisation mode (EI); a scan range of $100-400 \mathrm{~m} / \mathrm{z}$ and scan rate of two scans. $\mathrm{s}^{-1}$. A $30 \mathrm{~m}, 0.25 \mathrm{~mm}$ i.d., $0.25 \mu \mathrm{m}$ film thickness Zebron ZB-WAX (Phenomenex) was used with $1 \mathrm{~mL} \cdot \mathrm{min}^{-1} \mathrm{He}$ as carrier gas. The temperature gradient started at $165^{\circ} \mathrm{C}$, held for $10 \mathrm{~min}$, then ramped at $1.5^{\circ} \mathrm{C} \cdot \mathrm{min}^{-1}$ to $200^{\circ} \mathrm{C}$, then $20{ }^{\circ} \mathrm{C}$ to $250^{\circ} \mathrm{C}$ at $10^{\circ} \mathrm{C} \cdot \mathrm{min}^{-1}$ and held for $20 \mathrm{~min}$, giving a total run time of $65 \mathrm{~min}$. A $1 \mu \mathrm{L}$ 
injection was made with a 1:100 split and sample inlet temperature at $250{ }^{\circ} \mathrm{C}$. The Supelco 37 Component FAMEs mix standard (Supelco, Bellefonte, PA, USA) was used to identify the FAMEs of the grape seed oils. The FAMEs in samples were determined through their retention times and comparing spectra with the NIST 2011 Mass Spectral Library. The fatty acid quantities were expressed as relative percentages with respect to total fatty acid content $[22,23]$. Peak integration was carried out using Xcalibur ${ }^{\mathrm{TM}}$ software (Thermo Scientific $^{\mathrm{TM}}$, Waltham, MA, USA).

\subsection{HPLC-MS Analysis of Triacylglycerols}

In order to separate and identify the triacylglycerols (TAGs), a ballistic HPLC method has been applied [24]. The sample preparation consisted in an untreated grapeseed oil dilution; in particular, $0.5 \mu \mathrm{L}$ of each oil has been dissolved in $1.5 \mathrm{~mL}$ of methanol in an autosampler vial; there followed $5 \mathrm{~min}$ of sample sonication and, finally, $5 \mu \mathrm{L}$ of the diluted sample was injected. An Alliance 2695 (Waters) HPLC system equipped with autosampler, degasser and column heater coupled with a quadrupole time of flight (Waters Q-Tof Premier) mass spectrometer has been used. The compounds were separated by a Thermo Hypersil Gold $10 \mathrm{~cm}, 2.1 \mathrm{~mm} 1.9$ um particle size under the following conditions: column temperature, $20^{\circ} \mathrm{C}$; injected volume $5 \mu \mathrm{L}$. All samples have been injected in triplicate using a thermostatic autosampler maintained at $15{ }^{\circ} \mathrm{C}$. The HPLC analyses were carried out using a stepwise gradient program combining solvent $\mathrm{A}$ (methanol/water, 90/10, $v / v \%$ ), containing $0.2 \%$ acetic acid, and solvent B (methanol/n-hexane, $90 / 10 \mathrm{v} / \mathrm{v} \%$ ), containing $0.2 \%$ acetic acid. The elution gradient was the following: from 0 to $1 \mathrm{~min}, 100 \% \mathrm{~A}$ (flow rate $0.2 \mathrm{~mL} / \mathrm{min}$ ), from 1.01 to $10 \mathrm{~min}, 100 \% \mathrm{~B}$ (flow rate $0.2 \mathrm{~mL} / \mathrm{min}$ ), from 12 to $20 \mathrm{~min}$ the same percentage of solvent $B$ was maintained at flow $0.7 \mathrm{~mL} / \mathrm{min}$, from 20 to $21 \mathrm{~min} 100 \%$ $\mathrm{B}$ (flow rate $0.2 \mathrm{~mL} / \mathrm{min}$ ) and then from 21.01 to $31 \mathrm{~min}, 100 \%$ A (flow rate $0.2 \mathrm{~mL} / \mathrm{min}$ ). The MS experiments were performed on Q-Tof Premier using dynamic range enhancement (DRE) as acquisition mode that avoids MCP saturation keeping a good sensitivity. This allows to correctly quantify very abundant as well as trace-level compounds, providing results that were more reliable. Atmospheric pressure chemical ionization (APCI) has been used in positive mode under the following conditions: corona probe current, $4 \mu \mathrm{A}$; corona voltage, $3.6 \mathrm{KV}$; probe temperature, $450.0^{\circ} \mathrm{C}$; sampling cone, $19.0 \mathrm{~V}$; extraction cone, $4.3 \mathrm{~V}$; ion guide, $1.2 \mathrm{~V}$; source temperature $90^{\circ} \mathrm{C}$, cone gas $\mathrm{N} 2$, flow $50.0 \mathrm{~L} / \mathrm{h}$; desolvation gas $\mathrm{N} 2$, flow $300.0 \mathrm{~L} / \mathrm{h}$.

The triacylglycerols abundances and identification have also been validated performing an in-silico saponification using a freely distribute software (TAGSCHECK) [21].

\subsection{Statistical Analysis}

All analyses were performed in triplicate. Data are presented as mean \pm standard deviation. A $t$ test (confidence level 95\%) was performed to ascertain significant differences among fatty acid content in Soxhlet and cold pressing extraction.

\section{Results and Discussion}

The grape seed oil potential was explored by the characterization of fatty acid and triacylglycerol profile. Moreover, a classification of grape seed oil was also given.

\subsection{Grape Seed Oil Classification by Langual ${ }^{\mathrm{TM}}$ and Foodex 2}

The classification and coding food is a fundamental step to guarantee the standardization and harmonization of data and suitability to be inserted into Food Data Banks. There is a general consensus on the importance of the nomenclature, description and classification of foods. A coherent food description system is essential for comparing data from different databases, by favoring the exchanging from different organizations and countries. The classification and coding of grape seed oil by LanguaL ${ }^{\mathrm{TM}}$ and FoodEx 2 was applied for coding products in line with European data harmonization and standardization procedures. The generally recognized and commonly used description and classification systems are: 
LanguaL ${ }^{\mathrm{TM}}$ and FoodEx2. The Langua $\mathrm{L}^{\mathrm{TM}}$ is a multi-faceted thesaural system for describing systematically foods that has the requisite of flexibility in terms of food description, allowing description and categorization at different levels of detail [25]. FoodEx2 represents a system of flexible combination of classifications categories and descriptors based on a hierarchical system for different food safety-related domains [26-30].

Grape seed oil samples can be categorized by LanguaL ${ }^{\mathrm{TM}}$ as follows: LanguaL Facet: "PRODUCT TYPE" = A0806 (VEGETABLE FAT OR OIL (EUROFIR)); "FOOD SOURCE" = B1329 (Grape); "PART OF PLANT OR ANIMAL" = C0307 (Seed oil); "PHYSICAL STATE, SHAPE OR FORM" = E0139 (Liquid, High Viscosity, With No Visible Particles); "TREATMENT APPLIED" = H0130 (Physically/chemically modified); "CONSUMER GROUP/DIETARY USE/LABEL CLAIM" = P0024 (Human consumer, no age specification).

By means of FoodeEx2, revision 2, they can be classified throughout the base term [A037F] = Grape seed oil, by indicating "The group includes any type of Grape seed oil. The part consumed/analyzed is by default a portion representing the eventual heterogeneity of this item" [28].

The type of oil processing can be detailed for grape seed oil obtained by Soxhlet by inserting the additional FACET [F28.A0C07] = Oil production - solvent based; indeed, the samples can be classified as [A037F\# F28.A0C07]: Grape seed oil, PROCESS = Oil production - solvent based.

The type of oil processing can be detailed for grape seed oil obtained by cold pressed extraction by inserting the additional FACET [F28.A0C06] = Oil production - mechanical cold. Indeed, these samples can be classified as [A037F\# F28.A0C06]: Grape seed oil, PROCESS $=$ Oil production - mechanical cold .

\subsection{Fatty Acids Profile}

One of the most significant advances that have been made understands the importance of dietary fatty acids for human health.

Soxhlet oil extraction had a higher yield, for both red and white seed grapes. In fact, Soxhlet extraction of red seed grapes gave a yield of $8.1 \mathrm{~g} / 100 \mathrm{~g}$ and $7.6 \mathrm{~g} / 100 \mathrm{~g}$ for the cold press; on the other hand, for white seed grapes, the yield was $6.8 \mathrm{~g} / 100 \mathrm{~g}$ and $5.5 \mathrm{~g} / 100 \mathrm{~g}$, respectively.

Results showed that grape seeds oils contain a high percentage of linoleic acid methyl ester (67.2-71.1\%), oleic acid methyl ester (16.62-20.4\%), palmitic acid methyl ester $(7.9-8.88 \%)$ and stearic acid methyl ester (3.26-4.33\%). The percentage of minor FAME was $(0.27 \%) \alpha$-linolenic acid methyl ester but only in the cold pressed extraction procedure (Figure S1).

Qualitative and quantitative analysis of the methyl esters of fatty acids showed the presence of four main FAMEs and one minor FAME of the studied seed oil of Vitis vinifera as shown in Table 1.

According to the results, oil samples contained saturated fatty acids (11.36-12.4\%), monounsaturated fatty acids $(16.62-20.4 \%)$ and polyunsaturated fatty acids $(67.2-71.17 \%)$. Irrespective of extraction type (Soxhlet or cold pressed) or grape variety (red vs. white), four fatty acids were determined in all samples. Only in cold pressed red grape seed was a low percentage of $\alpha$-linolenic acid detected.

Among saturated fatty acids, palmitic acid was the predominant one (7.90-8.88\%) together with stearic acid (3.26-4.33\%). In the investigate samples, oleic acid was in a range $16.62-20.4 \%$ and linoleic acid was the most abundant polyunsaturated fatty acid accounting for $67.2-71.15 \%$ of all present total fatty acids. 
Table 1. Relative percentages of fatty acid profiles (percent of total fatty acid content) analyses and in silico saponification procedure. The values in parentheses represent the standard deviation. Data are expressed as mean $(n=3)$, SFA, MUFA and PUFA stand for saturated, mono unsaturated and poly unsaturated fatty acids, respectively. Means within the same row with different superscripts letters $(\mathrm{a}-\mathrm{c})$ are significantly different $(p<0.05)$. In Tables S1 and S2 are reported "one way ANOVA test" on FAMEs and in silico saponification data. nd = not detectable.

\begin{tabular}{|c|c|c|c|c|c|c|}
\hline \multirow{4}{*}{\multicolumn{2}{|c|}{ Fatty Acid }} & \multirow{4}{*}{ Rt (min) } & \multicolumn{4}{|c|}{ Oil Extraction Procedure } \\
\hline & & & \multicolumn{2}{|c|}{ Cold Pressed } & \multicolumn{2}{|c|}{ Soxhlet } \\
\hline & & & \multicolumn{4}{|c|}{$\%$ As Relative Percentage $( \pm S D)$} \\
\hline & & & $\begin{array}{l}\text { Red Grape } \\
\text { Seeds }\end{array}$ & $\begin{array}{c}\text { White Grape } \\
\text { Seeds }\end{array}$ & $\begin{array}{c}\text { Red Grape } \\
\text { Seeds }\end{array}$ & $\begin{array}{c}\text { White Grape } \\
\text { Seeds }\end{array}$ \\
\hline C16:0 & Palmitic acid & 20.09 & $8.88( \pm 0.02) \mathrm{a}$ & $8.2( \pm 0.2) c$ & $8.1( \pm 0.1) \mathrm{a}$ & $7.9( \pm 0.1) \mathrm{c}$ \\
\hline C18:0 & Stearic acid & 37.31 & $3.33( \pm 0.03) c$ & $4.2( \pm 0.5) \mathrm{c}$ & $3.26( \pm 0.07) \mathrm{c}$ & $4.33( \pm 0.01) \mathrm{c}$ \\
\hline C18: $\Delta 9$ & Oleic acid & 38.83 & $16.62( \pm 0.08) b$ & $20.4( \pm 0.2) c$ & $17.5( \pm 0.2) b$ & $19.8( \pm 0.6) c$ \\
\hline $\mathrm{C} 13: 2 \Delta 9,12$ & Linoleic acid & 41.82 & $70.9( \pm 0.7) \mathrm{c}$ & $67.2( \pm 0.2) c$ & $71.1( \pm 0.9) c$ & $68.0( \pm 0.9) \mathrm{c}$ \\
\hline $\mathrm{C} 18: 3 \Delta 9,12,15$ & $\alpha$-Linolenic acid & 45.16 & $0.27( \pm 0.01)$ a & nd & nd, a & nd \\
\hline & SFA & & $12.21( \pm 0.2)$ & $12.4( \pm 0.4)$ & $11.36( \pm 0.5)$ & $12.23( \pm 0.5)$ \\
\hline & MUFA & & $16.62( \pm 0.8)$ & $20.4( \pm 0.7)$ & $17.5( \pm 0.8)$ & $19.8( \pm 0.2)$ \\
\hline & PUFA & & $71.17( \pm 0.1)$ & $67.2( \pm 0.9)$ & $71.1( \pm 0.9)$ & $68.0( \pm 0.7)$ \\
\hline \multicolumn{7}{|c|}{ In silico saponification } \\
\hline C16:0 & Palmitic acid & & $9.0( \pm 0.1)$ & $8.4( \pm 0.3)$ & $9.61( \pm 0.05)$ & $7.13( \pm 0.02)$ \\
\hline C18:0 & Stearic acid & & $4.6( \pm 0.05)$ & $3.7( \pm 0.1)$ & $1.56( \pm 0.01)$ & $3.23( \pm 0.05)$ \\
\hline $\mathrm{C} 18: \Delta 9$ & Oleic acid & & $16.3( \pm 0.2)$ & $15.6( \pm 0.09)$ & $16.4( \pm 0.05)$ & $13.9( \pm 0.1)$ \\
\hline $\mathrm{C} 13: 2 \Delta 9,12$ & Linoleic acid & & $70.000( \pm 0.002)$ & $72.23( \pm 0.05)$ & $72.30( \pm 0.07)$ & $75.61( \pm 0.09)$ \\
\hline $\mathrm{C} 18: 3 \Delta 9,12,15$ & $\alpha$-Linolenic acid & & $0.063( \pm 0.004)$ & nd & $0.164( \pm 0.001)$ & nd \\
\hline
\end{tabular}

a: Significantly higher in cold pressed and lower in Soxhlet; b: significantly lower in cold pressed and higher in Soxhlet; c: no significant differences.

Previously, many authors have studied the fatty acid composition of triglycerides contained in grape seed oils obtained with a different extraction method, of various cultivar and geographic origins $[13,31,32]$. Beveridge et al. [31] reported relative percentages of linoleic acid in a range between 66.8 and $73.6 \%$ in seven distinct seed oils obtained from as many grape varieties. Oleic acid was between $12 \%$ and $19 \%$, while the saturated palmitic and stearic fatty acids were $6.28-8.62 \%$ and $3.60-5.26 \%$, respectively [31].

GC-MS analysis of fatty acids in grape seed oil was also performed by Alfekaik and AL-Hilfi [33]. In addition to other fatty acids found in small quantities (less than $0.3 \%$ ), linoleic acid (59.97\%), oleic acid (16.98\%), palmitic acid (12.09\%) and stearic acid $(8.85 \%)$ were shown among the main FAMEs. These values are in general in agreement with those reported by Dimíc et al. [9] as determined in grape seed oil or other matrices like in pumkin seed oil [34]. El-Shami [35] reported higher oleic acid contents in Egyptian grape seeds. Crews et al. [13] showed different proportions of fatty acids present in grape seed oils from France, Italy and Spain. It is also reassuring that the FAMEs percentages obtained from the in-silico saponification were practically superimposable with results obtained from traditional analysis. This confirms that the following TAGs analysis is reliable in both TAG assignments and relative abundances. In addition, it further confirms that it would be possible to reduce the sample analysis time to a very simple HPLC/MS TAGs determination.

\subsection{Triacylglycerols Profile}

TAGs analysis has proven a useful tool to characterize and discriminate vegetable oils $[21,24,36]$. Representing the major components of the oily matrices, their discrimination and quantitation allows one to evidence subtle differences among the matrices investigated. It has been reported that relying on TAGs qualitative and quantitative data could be a successful approach to individuate alimentary frauds, as illegal mixing of olive oil with lesser seed oils [36], or to distinguish among oils obtained from the same vegetable species 
but belonging to differing cultivars [22]. To enrich the still scarce information on grapeseed oils, it appeared appropriate to gather further TAGs information and add our findings to the actual literature base of data.

To achieve a TAGs characterization of grape seed oils, we adopted the HPLC/HRMS approach, using an APCI source that guarantees a good sensitivity and a good linearity for quantitation purposes. The adoption of a ballistic HPLC method allowed reducing of sample preparation to a simple dilution in methanol of the grape seed oils. The HPLC-MS analysis revealed that cold pressed grape seed oils consist of six abundant TAG species: LLL (trilinoleoyl glycerol), OLL (dilinoleoyl-oleoyl glycerol), LLP (dilinoleoyl-pailmityl glycerol), and, to a lesser extent, SLL (stearoyl-oleoyl-palmitoyl glycerol), LOP (linoleicoleoyl-palmitoyl glycerol) and OLO (oleoyl linoleic-oleoyl glycerol). The complete TAGs percent composition is reported in Table 2.

Table 2. Triacylglycerols (TAGs) content in red and white grape seed oils (relative percentage) by cold pressed and Soxhlet extraction.

\begin{tabular}{|c|c|c|c|c|c|c|c|c|c|}
\hline & & & & & TAGs & tive $\%$ & & & \\
\hline \multirow{8}{*}{$\begin{array}{l}\text { Cold pressed } \\
\text { extraction }\end{array}$} & \multirow{4}{*}{ White grape seeds } & OOMo & LLLn & LLL & OLL & LOP & $\mathrm{AOO}$ & SLL & OLO \\
\hline & & 0.4 & 0.1 & 34.8 & 21.9 & 5.6 & 0.2 & 9.0 & 5.7 \\
\hline & & $\mathrm{SOO}$ & SLO & OLMa & OOP & POPo & LLMa & LLP & LLPo \\
\hline & & 0.4 & 1.6 & 0.3 & 1.1 & 1.3 & 0.1 & 17.0 & 0.4 \\
\hline & \multirow{4}{*}{ Red grape seeds } & OOMo & LLLn & LLL & OLL & LOP & $\mathrm{AOO}$ & SLL & OLO \\
\hline & & 0.6 & 0.2 & 31.7 & 20.8 & 6.1 & 0.2 & 10.3 & 5.2 \\
\hline & & $\mathrm{SOO}$ & $\mathrm{SLO}$ & OLMa & OOP & POPo & LLMa & LLP & LLPo \\
\hline & & 0.7 & 2.7 & 0.2 & 1.9 & 1.4 & 0.3 & 17.2 & 0.4 \\
\hline \multirow{8}{*}{$\begin{array}{l}\text { Soxhlet } \\
\text { extraction }\end{array}$} & \multirow{4}{*}{ White grape seeds } & OOMo & LLLn & LLL & OLL & LOP & $\mathrm{AOO}$ & SLL & OLO \\
\hline & & 0.3 & 0.1 & 35.5 & 20.1 & 5.8 & 0.2 & 9.1 & 5.6 \\
\hline & & $\mathrm{SOO}$ & $\mathrm{SLO}$ & OLMa & OOP & POPo & LLMa & LLP & LLPo \\
\hline & & 0.4 & 1.7 & 0.3 & 1.3 & 1.2 & 0.1 & 17.6 & 0.4 \\
\hline & \multirow{4}{*}{ Red grape seeds } & OOMo & LLLn & LLL & OLL & LOP & $\mathrm{AOO}$ & SLL & OLO \\
\hline & & 0.5 & 0.2 & 31.5 & 21.1 & 6.2 & 0.3 & 10.1 & 5.1 \\
\hline & & $\mathrm{SOO}$ & SLO & OLMa & OOP & POPo & LLMa & LLP & LLPo \\
\hline & & 0.8 & 2.7 & 0.2 & 1.8 & 1.4 & 0.3 & 17.3 & 0.4 \\
\hline
\end{tabular}

The following abbreviations are used: A Arachidic acid; B behenic acid; G gadoleic acid; L linoleic acid; Ln linolenic acid; M myristic acid; Ma Margaric acid; Mo heptadecenoic acid; O oleic acid; P palmitic acid; Po palmitoleic acid; S stearic acid.

These results are qualitatively and quantitatively in excellent agreement with the literature data obtained with different analytical methodologies [37-39].

In our case, the most prominent components were detected at $\mathrm{m} / \mathrm{z} 879.741,881.757$ and 855.741 , identified on the basis of their accurate mass and of the corresponding diglyceridic fragments, and consist of trilinoleoyl glycerol (C54: 6, LLL) and dilinoleoyl-oleoyl glycerol (C54: 5, LLO) and dilinoleoyl-pailmityl glycerol (C52: 4, LLP), respectively (Figure 1). 


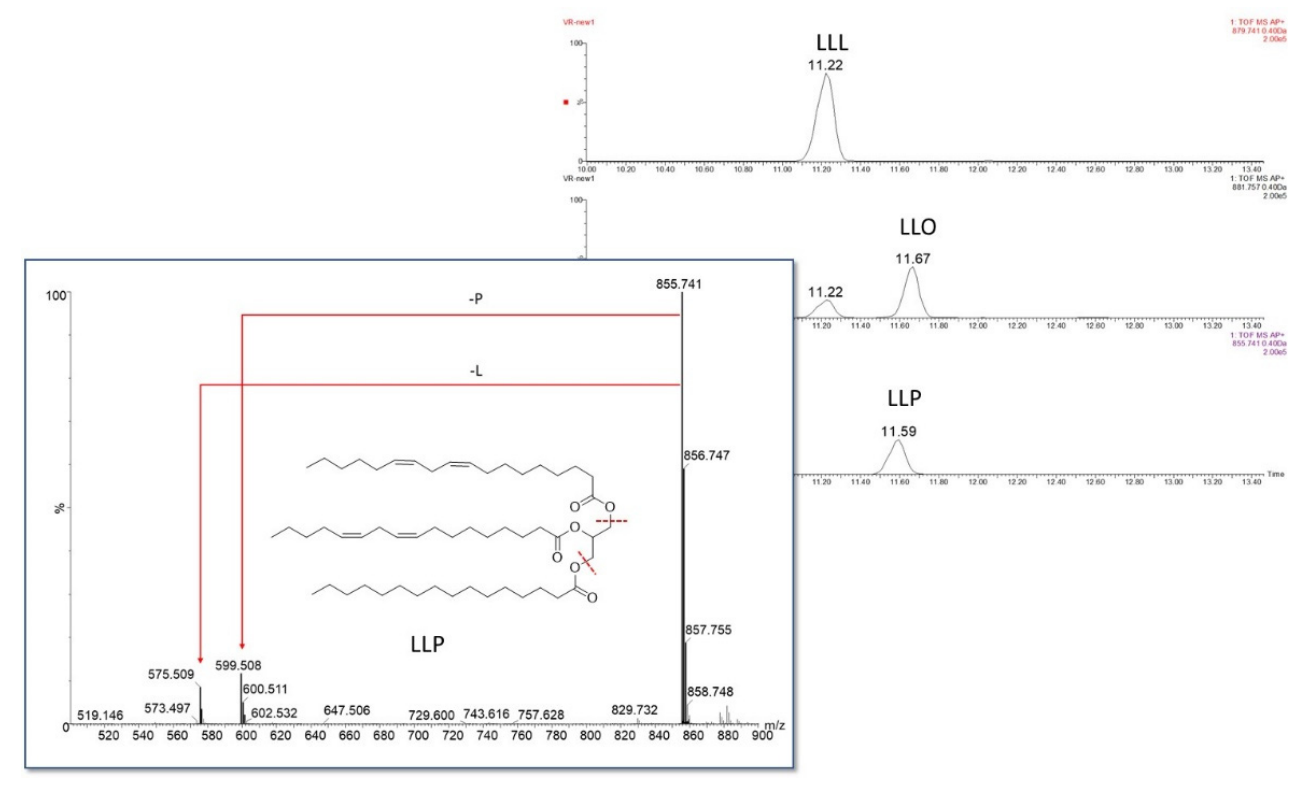

Figure 1. Chromatograms of the most prominent TAGs: LLL (trilinoleoyl glycerol), LLO (dilinoleoyloleoyl glycerol) and LLP (dilinoleoyl-pailmityl glycerol).

The comparison between grape seeds from white and red grapes showed only marginal differences in the relative abundances of the most abundant homologous TAG; more evident differences are observed for the less abundant TAGs, even if, in this case, the measurements are proportionally more susceptible to quantitation errors. Sensitive variations in the percentages of TAGs were not observed even in the samples obtained by different extraction method. As aforesaid, to corroborate TAGs analysis results, a cross validation procedure has been performed, comparing the FAMEs percentages obtained from the in-silico saponification with results obtained from the traditional analysis. To obtain FAMES data form TAGs determination is not a recently developed approach [40] but more recent [24] is the availability of simple free software to accomplish such a task, making the process reliable and straightforward. A proper in-silico FAMEs quantitation is possible only when both TAGs assignments and relative abundances have been correctly determined. In addition, the latter determination requires one to take into consideration also the differing ionization efficiencies that characterize each TAG, that are widely reported in the literature, and implemented in the latest version of the free TAGSCHECK software [24].

\section{Conclusions}

The correct management of the biomass requires the development of synergies between the various production sectors. The full exploitation of agri-food waste requires adequately differentiated and distributed supply chains, able to guarantee the cascade use, such as, for example, the preventive extraction of all products with high added value, also considering local differentiation and availability.

Grape seed oil representing an emerging vegetable fat, mainly used for culinary and pharmaceutical purposes as well as for various technical applications, was subject of the present investigation.

The results of the investigation of cold-pressed oil samples obtained from grapes grown in the Sicilian vineyard showed good oil quality and pronounced seed oil profile differences for red and white grape varieties were not observed. Grape seeds oils contain high percentage of linoleic acid (over 70\%), followed by oleic acid (about 17\%), palmitic acid $(9 \%)$ and stearic acid (3\%). The HPLC-TOF MS/MS analysis of grape seed oils revealed abundant TAG species (LLL, OLL, LLP, and to a lesser extent SLL, LOP and OLO). As can be seen, there are no detectable differences in terms of TAGs and fatty acids in the oils obtained by Soxhlet extraction. 
Chemical composition of cold-pressed grape seed oils is highly appreciated for their nutritional properties and push towards the production of this type of oil in greater quantities. Certainly, future analyses concerning the determination of other components present and the eventual evaluation of the oil composition variation after storage will be necessary.

Supplementary Materials: The following are available online at https:/ /www.mdpi.com/article/ 10.3390/su132313038/s1, Figure S1: FAMEs chromatogram related to the white grape seeds oil. Table S1: one way ANOVA on FAMEs. Table S2: one way ANOVA on in silico saponification data.

Author Contributions: Conceptualization, A.D., V.A., M.V., V.D.S. and D.B.; methodology, M.M., V.D.S. and D.B.; software, A.D.; validation, A.D., D.B., S.I. and V.D.S.; formal analysis, V.D.S., C.B., M.M. and D.B.; investigation, C.B. and A.F.; resources, V.A. and M.V.; data curation D.B and V.D.S.; writing—original draft preparation, A.S., A.D., M.L., M.V., D.B. and V.D.S.; writing—review and editing, V.A., M.V., C.B., A.S. and D.B.; supervision, V.A. and V.D.S.; project administration, V.A.; funding acquisition, V.A. All authors have read and agreed to the published version of the manuscript.

Funding: This study, was partially funded by the Project "CoSMetici dalla fiLiera vitIviNicola bioloGica-SMILING, PO FESR Azione 1.1.5-2017-NAZ-0246 CUP: G18I17000160007.

Data Availability Statement: The data presented in this study are available on request from the corresponding author.

Conflicts of Interest: The authors declare no conflict of interest.

\section{References}

1. Lin, C.S.K.; Koutinas, A.A.; Stamatelatou, K.; Mubofu, E.B.; Matharu, A.S.; Kopsahelis, N.; Pfaltzgraff, L.A.; Clark, J.H.; Panikolaou, S.; Kwan, T.H.; et al. Current and future trends in food waste valorization for the production of chemicals, materials and fuels: A global perspective. Biofuels Bioprod. Bioref. 2014, 8, 686-715. [CrossRef]

2. Varzakas, T.; Zakynthinos, G.; Verpoort, F. Plant food residues as a source of nutraceuticals and functional foods. Foods 2016, 5, 88. [CrossRef]

3. Kumar, H.; Yadav, A.N.; Kumar, V.; Vyas, P.; Dhaliwal, H.S. Food waste: A potential bioresource for extraction of nutraceuticals and bioactive compounds. Bioresour. Bioprocess. 2017, 4, 18. [CrossRef]

4. Lucarini, M.; Durazzo, A.; Romani, A.; Campo, M.; Lombardi-Boccia, G.; Cecchini, F. Bio-Based Compounds from Grape Seeds: A Biorefinery Approach. Molecules 2018, 23, 1888. [CrossRef]

5. Lucarini, M.; Durazzo, A.; Kiefer, J.; Santini, A.; Lombardi-Boccia, G.; Souto, E.B.; Romani, A.; Lampe, A.; Ferrari Nicoli, S.; Gabrielli, P.; et al. Grape Seeds: Chromatographic profile of fatty acids and phenolic compounds and qualitative analysis by FTIR-ATR Spectroscopy. Foods 2019, 9, 10. [CrossRef] [PubMed]

6. OIV: Organisation Internationale de la vigne et du vin. Global Economic Vitiviniculture Data. 2021. Available online: http: //www.oiv.int/public/medias/5681/en-communiqu-depresse-octobre-017.pdf (accessed on 3 September 2021).

7. Sotiropoulou, E.I.; Varelas, V.; Liouni, M.; Nerantzis, E.T. Grape Seed Oil: From a Winery Waste to a Value Added Cosmetic Product-a Review. 2015. Available online: https://www.researchgate.net/publication/312578959 (accessed on 3 November 2021).

8. Shinagawa, F.B.; De Santana, F.C.; Torres, L.R.O.; Mancini-Filho, J. Grape seed oil: A potential functional food? Food Sci. Technol. 2015, 35, 399-406. [CrossRef]

9. Dimić, I.; Teslić, N.; Putnik, P.; Bursać Kovačević, D.; Zeković, Z.; Šojić, B.; Mrkonjić, Ž.; Čolović, D.; Montesano, D.; Pavlić, B. Innovative and Conventional Valorizations of Grape Seeds from Winery By-Products as Sustainable Source of Lipophilic. Antioxidants 2020, 9, 568. [CrossRef]

10. Tobar, P.; Moure, A.; Soto, C.; Chamy, R.; Zúñiga, M.E. Winery solid residue revalorization into oil and antioxidant with nutraceutical properties by an enzyme assisted process. Water Sci. Technol. 2005, 51, 47-52. [CrossRef] [PubMed]

11. Karaman, S.; Karasu, S.; Tornuk, F.; Toker, O.S.; Geçgel, Ü.; Sagdic, O.; Ozcan, N.; Gül, O. Recovery Potential of Cold Press By-products Obtained from the Edible Oil Industry: Physicochemical, Bioactive, and Antimicrobial Properties. J. Agric. Food Chem. 2015, 63, 2305-2313. [CrossRef]

12. Cicero, N.; Albergamo, A.; Salvo, A.; Bua, G.D.; Bartolomeo, G.; Mangano, V.; Rotondo, A.; Di Stefano, V.; Di Bella, G.; Dugo, G. Chemical characterization of a variety of cold-pressed gourmet oils available on the Brazilian market. Food Res. Int. 2018, 109, 517-525. [CrossRef]

13. Crews, C.; Hough, P.; Godward, J.; Brereton, P.; Lees, M.; Guiet, S.; Winkelmann, W. Quantitation of the main constituents of some authentic grape-seed oils of different origin. J. Agric. Food Chem. 2006, 54, 6261-6265. [CrossRef]

14. Tangolar, S.G.; Özogul, Y.; Tangolar, S.; Torun, A. Evaluation of fatty acid profiles and mineral content of grape seed oil of some grape genotypes. Int. J. Food Sci. Nutr. 2009, 60, 32-39. [CrossRef] 
15. Bada, J.C.; León-Camacho, M.; Copovi, P.; Alonso, L. Characterization of grape seed oil from wines with protected denomination of origin (PDO) from Spain. Grasas Aceites 2015, 66, e085.

16. Sabir, A.; Unver, A.; Kara, Z. The fatty acid and tocopherol constituents of the seed oil extracted from 21 grape varieties (Vitis spp.). J. Sci. Food Agric. 2012, 92, 1982-1987. [CrossRef] [PubMed]

17. Calder, P.C. Fatty acids and inflammation: The cutting edge between food and pharma. Eur. J. Pharmacol. 2011, 668, 50-58. [CrossRef]

18. Raphael, W.; Sordillo, L.M. Dietary polyunsaturated fatty acids and inflammation: The role of phospholipid biosynthesis. Int. J. Mol. Sci. 2013, 14, 21167-21188. [CrossRef] [PubMed]

19. Bialek, A.; Czerwonka, M.; Bialek, M.; Lepionka, T.; Kaszperuk, K.; Banaszkiewicz, T.; Tokarz, A. Influence of Pomegranate Seed Oil and Grape Seed Oil on Cholesterol Content and Fatty Acids Profile in Livers of Chickens. Acta Pol. Pharm. 2017, 74, 624-632.

20. Sharifi, M.; Bashtani, M.; Naserian, A.A.; Farhangfar, H.; Emami, A. The effect of grapeseed oil on performance, rumen fermentation, antioxidant status and subcutaneous adipose fatty acid profile in lambs. J. Anim. Physiol. Anim. Nutr. 2018, 102, 157-165. [CrossRef]

21. Indelicato, S.; Bongiorno, D.; Ceraulo, L.; Emmanuello, C.; Mazzotti, F.; Siciliano, C.; Piazzese, D. One-Pot Analysis: A New Integrated Methodology for Determination of TAG and FA Determination through LC/MS and in-silico Saponification. Food Anal. Methods 2018, 11, 873-882. [CrossRef]

22. Grilo, F.; Novara, M.E.; D’Oca, M.C.; Rubino, S.; Lo Bianco, R.; Di Stefano, V. Quality evaluation of extra-virgin olive oils from Sicilian genotypes grown in a high-density system. Int. J. Food Sci. Nutr. 2020, 71, 397-409. [CrossRef] [PubMed]

23. Di Stefano, V.; Melilli, M.G. Effect of storage on quality parameters and phenolic content of Italian extra-virgin olive oils. Nat. Prod. Res. 2020, 34, 78-86. [CrossRef]

24. Agozzino, P.; Avellone, G.; Bongiorno, D.; Ceraulo, L.; Indelicato, S.; Indelicato, S.; Vèkey, K. Determination of the cultivar and aging of Sicilian olive oils using HPLC-MS and linear discriminant analysis. J. Mass Spectrom. 2010, 45, 989-995. [CrossRef] [PubMed]

25. Møller, A.; Ireland, J. LanguaL ${ }^{\mathrm{TM}}$ 2017-the LanguaL ${ }^{\mathrm{TM}}$ thesaurus technical report. 2018, Danish Food Informatics (ISBN 97887-92125-26-2). Available online: https://www.langual.org/download/LanguaL2017/LanguaL\%202017\%20Thesaurus.pdf (accessed on 21 November 2021).

26. European Food Safety Authority (EFSA). Training on FoodEx2. EFSA Support. Publ. 2018, 15, 1437E.

27. European Food Safety Authority (EFSA); Ioannidou, S.; Nikolic, M.; Gibin, D. FoodEx2 maintenance 2016-2018. EFSA Support. Publ. 2018, 16, 1584E.

28. European Food Safety Authority. The food classification and description system FoodEx2 (revision 2). EFSA Support. Publ. 2015, 12, 804E.

29. European Food Safety Authority; Vernazza, F.; Avon, V. FoodEx2 Browser-User's guide. EFSA Support. Publ. 2017, 14, 1291E.

30. European Food Safety Authority; Vernazza, F.; Avon, V. FoodEx2 Browser enhancement. EFSA Support. Publ. 2017, 14, 1292E.

31. Beveridge, T.H.J.; Girard, B.; Kopp, T.; Drover, J.C.G. Yield and composition of grape seed oils extracted by supercritical carbon dioxide and petroleum ether: Varietal effects. J. Agric. Food Chem. 2005, 53, 1799-1804. [CrossRef] [PubMed]

32. Martin, M.E.; Grao-Cruces, E.; Millan-Linares, M.C.; Montserrat-de la Paz, S. Grape (Vitis vinifera L.) Seed Oil: A Functional Food from the Winemaking Industry. Foods 2020, 9, 1360. [CrossRef]

33. Alfekaik, D.F.; AL-Hilfi, S.A. Fatty acids composition by (GC-MS) and most important physical chemicals parameters of seed oil pomegranate and grape seeds. J. Biol. Agric. Healthc. 2016, 6, 25-32.

34. Montesano, D.; Blasi, F.; Simonetti, M.S.; Santini, A.; Cossignani, L. Chemical and Nutritional Characterization of Seed Oil from Cucurbita maxima L. (var. Berrettina) Pumpkin. Foods 2018, 7, 30. [CrossRef]

35. El-Shami, S.M.; El-Mallah, M.H.; Mohamed, S.S. Studies on the lipid constituents of grape seeds recovered from pomace resulting from white grape processing. Grasas Aceites 1992, 43, 157-160. [CrossRef]

36. Indelicato, S.; Bongiorno, D.; Pitonzo, R.; Di Stefano, V.; Calabrese, V.; Indelicato, S.; Avellone, G. Triacylglycerols in edible oils: Determination, characterization, quantitation, chemometric approach and evaluation of adulterations. J. Chromatogr. A 2017, 1515, 1-16. [CrossRef]

37. Barron, L.J.R.; Celaa, M.V.; Santa-Maria, G.; Corzo, N. Determination of the triglyceride composition of grapes by HPLC. Chromatographia 1988, 25, 609-612. [CrossRef]

38. Bail, S.; Stuebiger, G.; Krist, S.; Unterweger, H.; Buchbauer, G. Characterisation of various grape seed oils by volatile compounds, triacylglycerol composition, total phenols and antioxidant capacity. Food Chem. 2008, 108, 1122-1132. [CrossRef]

39. De Marchi, F.; Seraglia, R.; Molin, L.; Traldi, P.; De Rosso, M.; Panighel, A.; Dalla Vedova, A.; Gardiman, M.; Giust, M.; Flamini, R. Seed oil triglyceride profiling of thirty-two hybrid grape varieties. J. Mass Spectrom. 2012, 47, 1113-1119. [CrossRef]

40. Byrdwell, W.C.; Emken, E.A.; Neff, W.E.; Adlof, R.O. Quantitative Analysis of Triglycerides Using Atmospheric Pressure Chemical Ionization-Mass Spectrometry. Lipids 1996, 31, 919-935. [CrossRef] 